\title{
Function of miR-152 as tumor suppressor in oral squamous cell carcinoma cells by targeting c-MET
}

\author{
MINGHE LI ${ }^{1}, \mathrm{ZHIHONG} \mathrm{LI}^{2}, \mathrm{XUE} \mathrm{WANG}^{3}, \mathrm{YUMEI} \mathrm{WANG}^{1}, \mathrm{CONG} \mathrm{ZHAO}^{4}$ and LEI WANG ${ }^{5}$ \\ ${ }^{1}$ Department of Oral and Maxillofacial Surgery, Hospital of Stomatology, Jilin University; \\ ${ }^{2}$ Department of Thoracic Surgery, The First Hospital of Jilin University, Changchun, Jilin 130021; \\ ${ }^{3}$ Department of Dentistry, The General Hospital of FAW, Changchun, Jilin 1300000; \\ Departments of ${ }^{4}$ Endodontics and ${ }^{5}$ Periodontology, Hospital of Stomatology, \\ Jilin University, Changchun, Jilin 130021, P.R. China \\ Received June 15, 2017; Accepted December 1, 20
}

DOI: 10.3892/or.2017.6157

\begin{abstract}
MicroRNA-152 (miR-152) has been reported to be involved in tumor development and progression in multiple cancers. However, the expression level, biological function and regulatory mechanisms of miR-152 in oral squamous cell carcinoma cells (OSCC) remain unclear. The aims of this study were therefore to investigate the role of miR-152 in OSCC and the relevant mechanism. It was found that miR-152 was downregulated in OSCC cell lines and tissues, and that decreased miR-152 was closely associated with lymph node metastasis, and patient survival rate. In vitro restoration of miR-152 significantly repressed cell proliferation, colony formation, migration and invasion of OSCC cells. Notably, cellular-mesenchymal to epithelial transition factor (c-MET) and its downstream signaling pathway (PI3K/AKT) was downregulated in OSCC cells by miR-152 through direct interactions with its $3^{\prime}$ untranslated region. Restoring c-MET expression attenuated miR-152-induced inhibitory effects in OSCC cells. In vivo study confirmed that restoration of miR-152 suppressed tumor growth in xenograft nude mice by repressing c-MET. In summary, the present study highlight miR-152 as a tumor suppressor in OSCC through direct targeting c-MET, rendering miR-152 a promising therapeutic target for oral squamous cell carcinoma.
\end{abstract}

\section{Introduction}

Oral squamous cell carcinoma cells (OSCC) is the most common oral cancer, and accounts for $\sim 90 \%$ total oral cancers (1). Despite considerable improvements in therapeutic

Correspondence to: Dr Lei Wang, Department of Periodontology, Hospital of Stomatology, Jilin University, Changchun, Jilin 130021, P.R. China

E-mail: wangleijluedu@126.com

Key words: oral squamous cell carcinoma cells, miR-152, c-MET, proliferation strategies for OSCC over the past two decades, the 5-year survival rate of OSCC has not improved significantly and its incidence continues to increase among young people and women $(2,3)$. Therefore, exploring novel molecular mechanisms involved in development and progression of OSCC is urgently needed for identifying novel and effective therapeutic targets.

MicroRNAs (miRNAs) are a class of highly conserved endogenous, single-stranded, small non-protein-coding RNAs comprising $\sim 18-25$ nucleotides that inhibit gene expression at the post-transcriptional level by directly binding to the 3'-untranslated region (3'UTR) of messenger RNAs (mRNAs) (4). Through repressing the target gene, miRNAs are able to regulate diverse biological processes, such as cell proliferation, cell migration, apoptosis, differentiation, development, immunity, and metabolism $(5,6)$. Importantly, miRNAs are dysregulated in various types of cancer as oncogenes or tumor suppressors $(7,8)$. For OSCC, number of miRNAs have been identified to be involved in progression and development of OSCC $(9,10)$, and to be used as diagnosis marker and therapy target.

MicroRNA-152 (miR-152) has been shown to be downregulated, and function as a tumor suppressor in many types of cancers, including gastric cancer (11), colorectal cancer (12), breast cancer (13), non-small lung cancer (14), cervical cancer (15), and prostate cancer (16). However, its potential role and mechanism in regulating OSCC carcinogenesis and progression remains unclear. This study aimed to reveal the exact role as well as the relevant mechanism of miR-152 in the regulation of OSCC cell proliferation and invasion.

\section{Materials and methods}

Tissue samples. Total of 40 paired fresh surgically resected OSCC tumors and matched adjacent normal tissues were obtained from OSCC patients who were diagnosed, treated, and followed-up at the Department of Oral and Maxillofacial Surgery, School and Hospital of Stomatology, Jilin University (Changchun, China). Fresh frozen tissues were immediately frozen in liquid nitrogen and stored in liquid nitrogen until RNA extraction. All samples were confirmed by pathological 
examinations. Written informed consent was obtained from all participants. This study was approved by the Ethics Committee of School and Hospital of Stomatology, Jilin University (Changchun, China). Patients who had received chemotherapy or radiotherapy prior to surgery were excluded. Clinical parameters, including pathological features and clinical stage, were retrospectively harvested and are presented in Table I.

Cell culture and transfection. The human normal oral keratinocytes (hNOKs) and four human OSCC cell lines, including Tca8113, OSCC-15, SCC-9, and SCC-25, were obtained from American Type Culture Collection (Manassas, VA, USA), and cultured in DMEM/Nutrient Mixture F12 (Hyclone, Logan, UT, USA) supplemented with $10 \%$ fetal bovine serum (FBS, Gibco, USA), $100 \mathrm{U} / \mathrm{ml}$ penicillin (Sigma-Aldrich, St. Louis, MO, USA), or $100 \mu \mathrm{g} / \mathrm{ml}$ streptomycin (Sigma-Aldrich) in a humidified incubator containing $5 \% \mathrm{CO}_{2}$ at $37^{\circ} \mathrm{C}$. The cells from passage 4 were used for all the experiments.

miR-152 mimic or corresponding negative control (miR-NC) was brought from GenePharma (Shanghai, China). The c-MET overexpression vector (pCDNA3.1-c-MET, lack of 3'UTR) was granted from Yue Zhao (Jilin University). Transfection was performed using the Lipofectamine ${ }^{\mathrm{TM}} 2000$ reagent (Invitrogen, Carlsbad, CA, USA) following the manufacturer's protocol.

Quantitative real-time PCR. Total RNA was extracted from the cultured cells or tissues using TRIzol (Invitrogen) according to the manufacturer's instructions. A dual-beam ultraviolet spectrophotometer (Eppendorf, Hamburg, Germany) were used to determine purity and concentration of total RNA. Then, total RNA was reverse transcribed to cDNA using a PrimeScript ${ }^{\circledR}$ RT reagent kit (Takara Biotechnology Co., Ltd., Dalin, China) or the miScript Reverse Transcription kit (Qiagen, Hilden, Germany). The PCR were performed using SYBR premix real-time PCR reagent (Takara) under an ABI7900 real-time PCR system (Applied Biosystems, Foster City, CA, USA). The primers for miR-152 and U6 were brought from Applied Biosystems. The primers for c-MET and GAPDH were used in this study as described previously (17). U6 and GAPDH were applied as internal controls for miR-152 and $c$-MET mRNA, respectively. Relative quantification was analyzed using the comparative $2^{-\Delta \Delta \mathrm{Ct}}$ method.

Cell proliferation assay. 3-(4,5-dimethylthiazol-2-yl)-2,5-diphenyl tetrazolium bromide (MTT) assay kit (Sigma-Aldrich) were used to measure cell proliferation. In brief, the transfected cells were seeded in a 96-well plate $\left(1 \times 10^{3}\right.$ cells/well) and cultured for 1-3 days. After incubated for 24, 48 and $72 \mathrm{~h}$ respectively, $25 \mu \mathrm{l}$ of MTT $(10 \mathrm{mg} / \mathrm{ml})$ was added to each well and cultured for $4 \mathrm{~h}$. Then, the supernatant was removed, and $150 \mu \mathrm{l}$ of dimethyl sulfoxide (DMSO, Sigma-Aldrich, St. Louis, MO, USA) was added to each well for $10 \mathrm{~min}$ at room temperature. The absorbance at $490 \mathrm{~nm}$ was detected with a microplate reader (Bio-Rad, Hercules, CA, USA).

Colony formation assay. Transfected cells were seeded in 6 -well plates (500 cells per well) and allowed to grow for 10 days to determine their colony-forming ability. After
Table I. Association of miR-152 expression with clinicopathological factors of 40 OSCC patients.

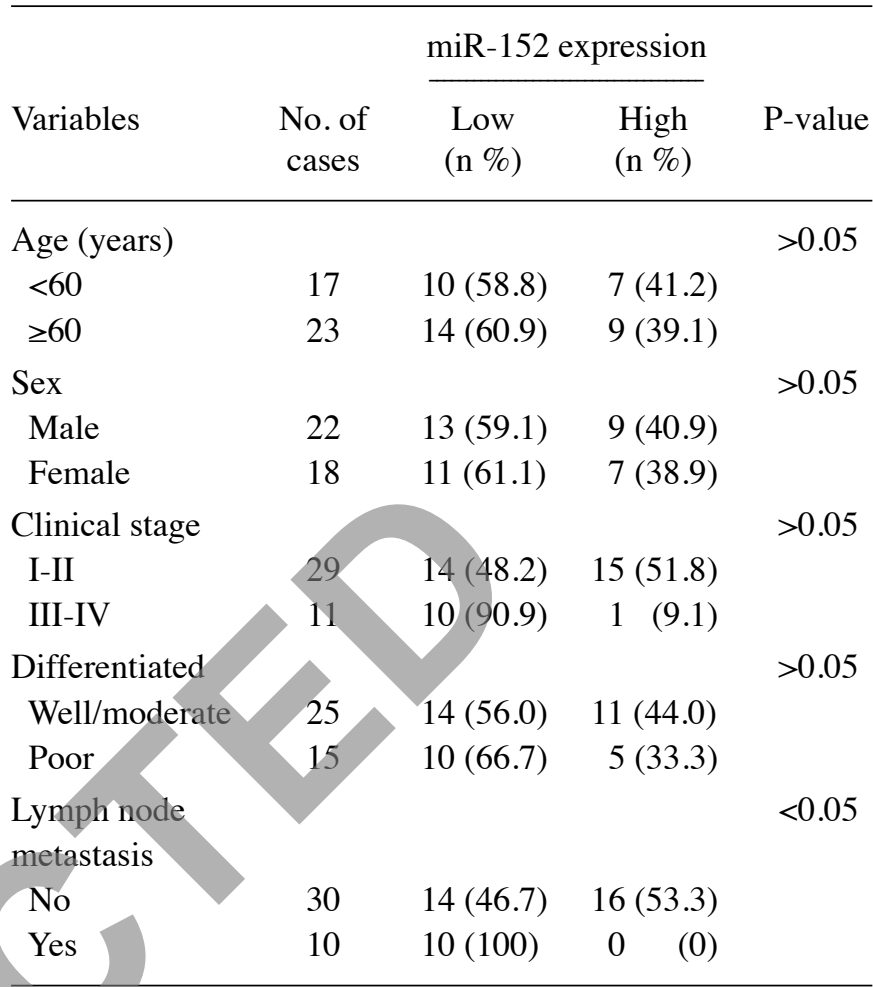

$70 \%$ ethanol fixation, the plate was stained with $0.1 \%$ crystal violet for $5 \mathrm{~min}$ (Sigma). Cell colonies were counted under a light microscope (Zeiss, Jena, Germany).

Migration and invasion assays. To determine cell migration, wound healing assay was performed when transfected cells grew to $100 \%$ confluence in 6-well plates. Wounds were created with a $10-\mu 1$ pipette tip to scratch the culture. After cells were rinsed with PBS, the medium was then added, and cultured for $24 \mathrm{~h}$ at $37^{\circ} \mathrm{C}$. At 0 and $24 \mathrm{~h}$ after the scratch, the cultures were examined and photographed under a light microscope (Zeiss).

For invasion assay, $1 \times 10^{5}$ transfected cells were seeded into the upper chambers with Matrigel ${ }^{\mathrm{TM}}$ Basement Membrane Matrix (BD Biosciences) in free serum. Medium with $20 \%$ FBS was added to the lower chambers to stimulate cell invasion. Twenty-four hours later, cells in the upper chambers were removed, while cells that migrated to the lower surface were fixed with $70 \%$ ethanol for 10 min and stained with $0.1 \%$ crystal violet (MedChemExpress, Princeton, NJ, USA) for $15 \mathrm{~min}$. The numbers of invaded cells were observed using a light microscope (magnification, x200, Zeiss) and counted in five randomly selected fields.

Luciferase reporter assay. miR-152 target gene was predicted by three publicly available algorithms: TargetScan, PicTar, and miRBase. c-MET was chosen as a target of miR-152. $c-M E T$ 3'UTR regions containing predicted miR-152 binding sites were amplified by PCR, and the production was subcloned into the pGL3 vector (Ambion, Austin, TX, USA), named as WT-c-MET-3'UTR. In addition, the binding sequence of c-MET 


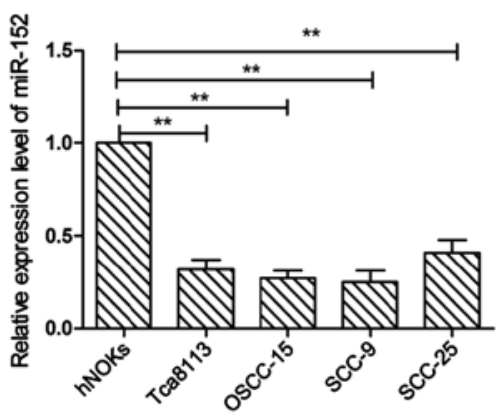

B

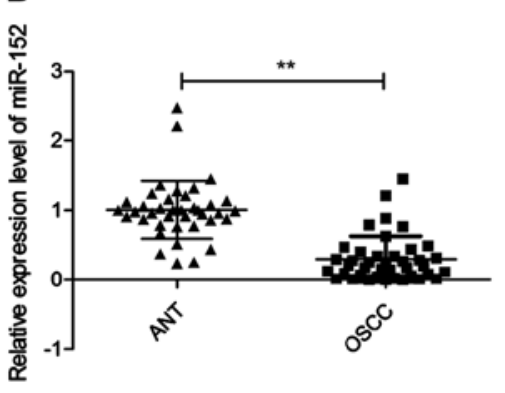

C

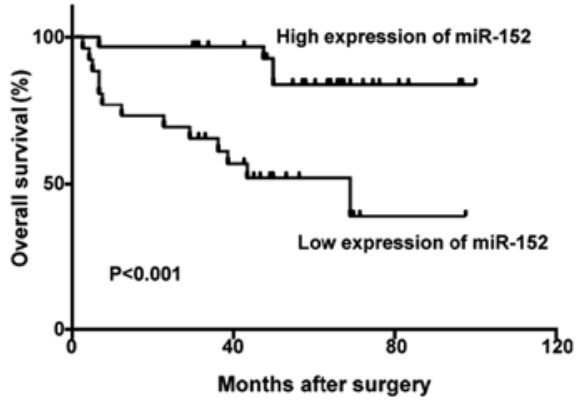

Figure 1. miR-152 is downregulated in both OSCC cells and clinical specimens. (A) Relative expression of miR-152 in the human normal oral keratinocytes (hNOKs) and the four human OSCC cell lines Tca8113, OSCC-15, SCC-9 and SCC-25. (B) Relative expression of miR-152 in 40 human OSCC tissues and adjacent normal tissues. (C) Kaplan-Meier survival curves of 40 OSCC patients divided by miR-152 expression. Log-rank test was used for survival analysis. The endogenous U6 RNA was used as the internal control. ${ }^{*} \mathrm{P}<0.05,{ }^{* *} \mathrm{P}<0.01$.

3'UTR was mutated using a Quik-Change ${ }^{\mathrm{TM}}$ Site-Directed Mutagenesis kit (Stratagene; Agilent Technologies, Inc., Santa Clara, CA, USA), and also cloned into pGL3 vector, named as: MUT-c-MET-3'UTR. For the luciferase reporter assay, SCC-9 cells were cotransfected with miR-152 mimic or miR-NC and WT-c-MET-3'UTR or MUT-c-MET-3'UTR reporter plasmid by Lipofectamine 2000. After $48 \mathrm{~h}$ cell transfection, luciferase activities were detected using a Dual-Luciferase ${ }^{\circledR}$ Reporter assay kit (Promega, Madison, WI, USA) according to the manufacturer's protocols. The results were presented as the ratio of firefly luciferase activity to renilla luciferase activity.

Western blotting. The total cell lysates were harvested using a detergent lysis buffer (Sigma-Aldrich). The protein concentration of each sample was measured using a BCA protein assay kit (Bio-Rad). The protein was separated on $10 \%$ SDS polyacrylamide gels, subsequently transferred to polyvinylidene difluoride (PVDF) membranes (Merck, Millipore, Germany). Then, the membrane were blocked with a 5\% skim milk solution and incubated with the following antibodies overnight at $4^{\circ} \mathrm{C}$ : anti-c-MET $(1: 1,000$, Santa Cruz Biotechnology, Inc., Santa Cruz, CA, USA), anti-AKT (1:2,000, Santa Cruz Biotechnology, Inc.) and anti-p-AKT (1:1,500, Santa Cruz Biotechnology, Inc.), anti-PI3K (1:2,000, Santa Cruz Biotechnology, Inc.) and anti-p-PI3K (1:1,000, Santa Cruz Biotechnology, Inc.), anti-GAPDH (1:5,000, Santa Cruz Biotechnology, Inc.). The membranes were then incubated with the corresponding secondary HRP-conjugated antibodies (1:5,000; Cell Signaling Technology, Inc., Danvers, MA, USA) for $2 \mathrm{~h}$ at room temperature. GAPDH was used as a loading control. Protein bands were observed by the enhanced chemiluminescence system (ECL kit, Millipore, Billerica, MA, USA) and were analyzed with the Bio-Rad ChemiDoc XRS system (Bio-Rad).

Mouse xenograft models. All experimental procedures were approved by the experimental animal ethics committee of Jilin University (Changchun, China). Twenty male BALB/c nude mice (6-week-old) were housed and maintained in a pathogen-free (SPF) environment. SCC-9 cells transfected with miR-152 mimic or miR-NC $\left(2 \times 10^{6}\right)$ were mixed with Matrigel (BD Lifesciences) and injected subcutaneously into the right flank of each animal ( $n=10$ each group), respectively. The tumor volume was measured once every five days until the mice were sacrificed using the following formula: tumor volume $(V)=\left(\right.$ length $\times$ width $\left.{ }^{2}\right) / 2$. The mice were sacrificed on day 30 after inoculation, then tumor tissues were stripped and weighted. The tumor xenografts were fixed and prepared for immunohistochemistry (IHC). IHC was performed as described previously (18)

Statistical analysis. Results are presented as mean \pm standard deviation (SD) from at least three independent experiments, and was analyzed using version 19 SPSS statistical software (SPSS, Chicago, IL, USA). The data were analyzed between two groups using Student's t-test or more than two groups using one-way analysis of variance (ANOVA). Spearman's correlation coefficient was used to assess correlations between miR-152 and $c$-MET. The survival ratio was analyzed by Kaplan-Meier plots. For all statistical analyses, P-values of $<0.05$ were considered to be statistically significant.

\section{Results}

miR-152 is downregulated in both OSCC cells and clinical specimens. In order to examine miR-152 expression levels, we first examined miR-152 expression in the human normal oral keratinocytes (hNOKs) and four human OSCC cell lines Tca8113, OSCC-15, SCC-9, and SCC-25. As shown in Fig. 1A, miR-152 expression was aberrantly downregulated in all OSCC cell lines, as compared to normal oral keratinocytes (hNOKs) $(\mathrm{P}<0.05)$. Next, we examined miR-152 expression in 40 pairs of OSCC tissues and matched adjacent normal tissues. We found that miR-152 expression level in OSCC tissues was significantly lower than those of their matched adjacent normal tissues $(\mathrm{P}<0.01)$. To further investigate the clinicopathological significance of miR-152 level in OSCC patients, based on the mean (0.29) of all samples, patients were divided into two subgroups: low miR-152 group $(<0.29$, 24 cases) and a high miR-152 group ( $>0.29,16$ cases). The results demonstrated that the decreased miR-152 expression was significantly associated with lymph node metastasis $(\mathrm{P}<0.05)$, but not with age, sex, clinical stage or differentiation (Table I, all $\mathrm{P}>0.05$ ). In addition, Kaplan-Meier analysis revealed that the OSCC patients with low miR-152 expression 

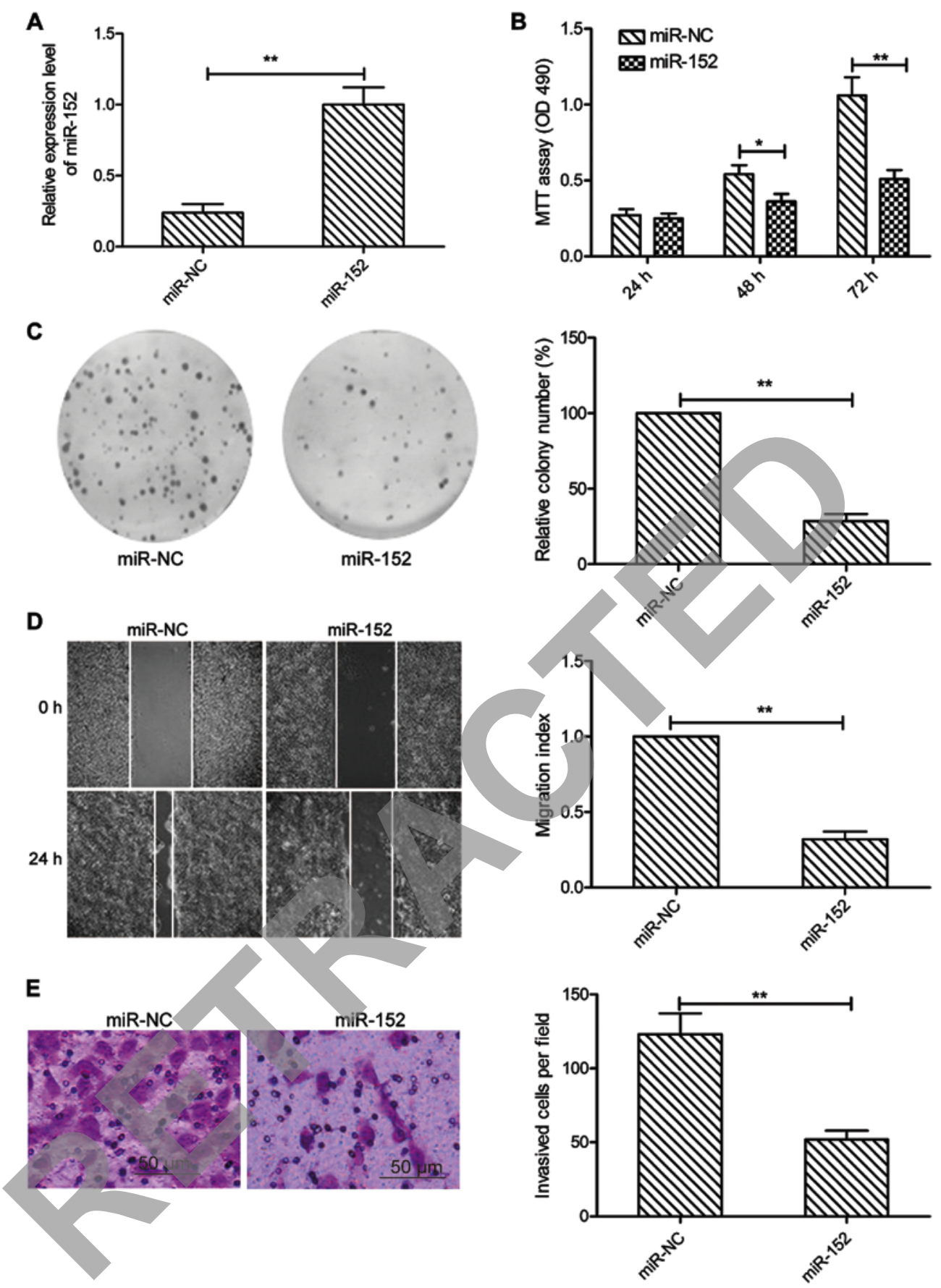

Figure 2. miR-152 inhibits proliferation, colony formation, migration and invasion of OSCC cells. (A) Relative expression of miR-152 was detected in SCC-9 cells transfected with miR-152 mimic or miR-NC. The endogenous U6 RNA was used as the internal control. (B-E) Cell proliferation (B), colony formation (C), migration (D) and invasion (E) were detected in SCC-9 cells transfected with miR-NC or miR-152 mimic. ${ }^{*} \mathrm{P}<0.05,{ }^{* *} \mathrm{P}<0.01$.

had poor survival rate (Fig. 1C). These results implied that miR-152 could serve as a prognostic marker and play a key role in OSCC progression.

miR-152 inhibits the proliferation, colony formation, migration, and invasion of OSCC cells. To explore the role of miR-152 in OSCC, we transfected miR-152 mimic or miR-NC mimic into SCC-9 cells, then cell proliferation, colony formation, migration and invasion were determined in the above cells. It was found that SCC-9 cells transfected with miR-152 mimic could significantly enhance miR-152 expression level compared to cells transfected with miR-NC
(Fig. 2A). MTT assay showed that cell viability was significantly reduced in miR-152-transfected cells compared with the miR-NC-transfected cells (Fig. 2B). Consistent with this result, miR-152 overexpression significantly decreased the colony formation of SCC-9 cells (Fig. 2C). Subsequently, we evaluated whether miR-152 was able to suppress the migration and invasion of OSCC cells by wound healing and Transwell invasion assays. Our results showed that overexpression of miR-152 caused a significantly suppression of cell migration (Fig. 2D), and invasion (Fig. 2E) capability in SCC-9 cells. These data implied that miR-152 might play a suppressive role in OSCC development. 
A

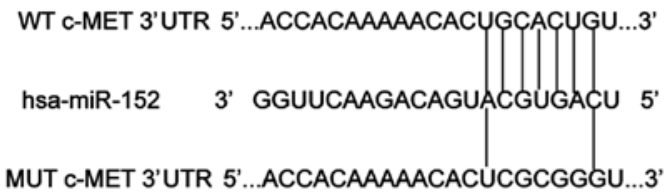

C

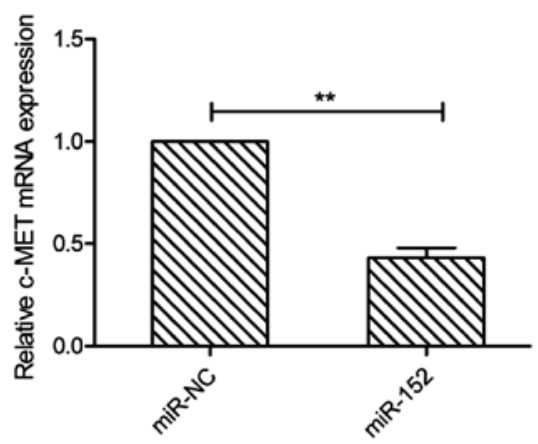

\section{B}

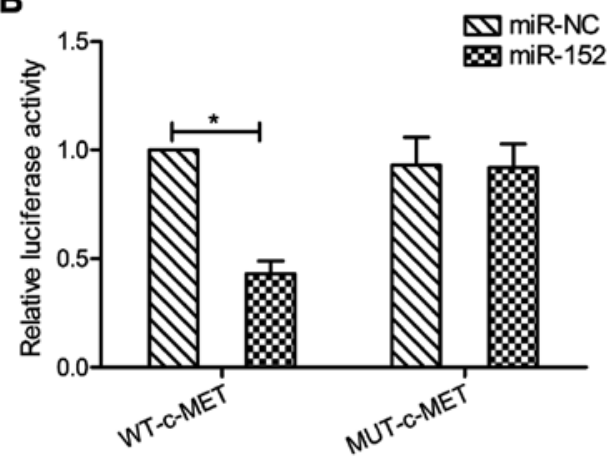

D

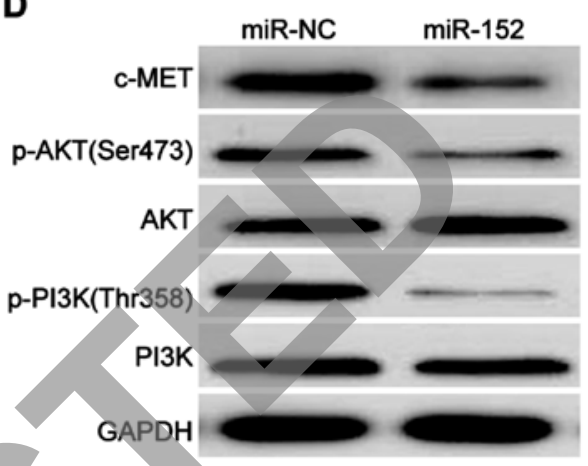

Figure 3. c-MET is a direct target of miR-152 in OSCC cells. (A) Schematic representation of the miR-152 putative binding sites in the 3'UTR of $c$-MET mRNA and the mutations introduced into the c-MET-3'UTR regions. (B) Relative luciferase activity in SCC9 cells co-transfection with WT or MUT-3'UTR c-MET reporter plasmids and miR-152 mimic or miR-NC. WT, wild-type; MUT, mutant-type. (C) Relative mRNA expression of $c$-MET was measured in SCC9 cells transfected with miR-152 mimic or miR-NC. GAPDH was used as the internal control.(D) c-MET, PI3K, p-PI3K, AKT and p-AKT protein expression were determined by western blotting in SCC9 cells transfected with miR-152 mimic or miR-NC. GAPDH was used as the internal control. "P $<0.05,{ }^{* * *} \mathrm{P}<0.01$.
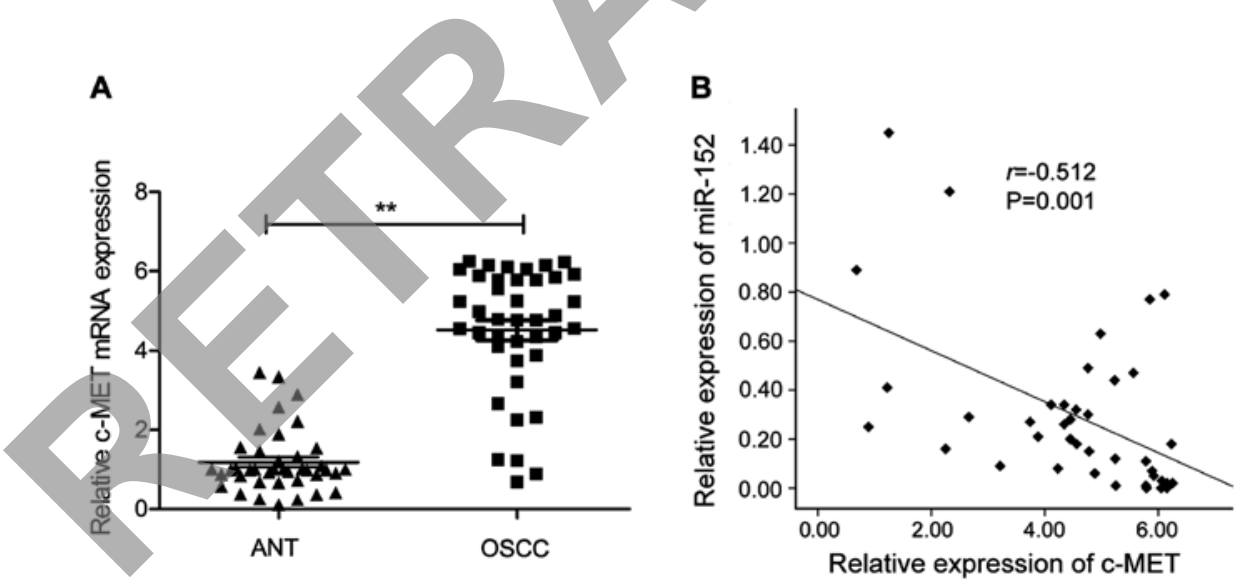

Figure 4. Inverse correlation between c-MET and miR-152 expression in OSCC tissues. (A) Relative mRNA expression of $c$-MET was measured in a cohort of 40 human OSCC tissues and adjacent normal tissues. GAPDH was used as the internal control. (B) Spearman's correlation analysis for the correlation of c-MET and miR-152 expression levels in patients with OSCC ( $\mathrm{n}=40 ; \mathrm{r}=-0.512 ; \mathrm{P}=0.001)$.

c-MET is a direct target of miR-152 in OSCC cells. miR-152 targets were predicted using the three algorithms: TargetScan, PicTar, and miRBase. A complementary sequence of miR-152 to the 3'UTR of $c$-MET mRNA is shown in Fig. 3A. The dual-luciferase reporter assay showed that that ectopic expression of miR-152 significantly decreased the luciferase activity of the WT-c-MET but not that of the MUT-c-MET-3'UTR in SCC-9 cells ( $\mathrm{P}>0.05$, Fig. 3B). More importantly, c-MET expression on mRNA and protein levels were both downregulated in SCC-9 cells transfected with miR-152 mimic compared those of cells transfected with miR-NC (Fig. 3C and D). In addition, our results also demonstrated that miR-152 significantly decreased $\mathrm{p}-\mathrm{PI} 3 \mathrm{~K}$ and $\mathrm{p}-\mathrm{AKT}$ protein expression, a downstream pathway of c-MET (Fig. 3D). These findings implied that miR-152 directly targeted $c-M E T$ in OSCC cells.

Inverse correlation between $c$-MET and miR-152 expression in OSCC tissues. We next applied qRT-PCR to examine the $c$-MET mRNA expression in 40 pairs of OSCC tissues and the corresponding adjacent normal tissues. As shown in Fig. 4A, $c$-MET mRNA expression level was significantly upregulated in OSCC tissues compared to the adjacent normal tissues. Moreover, through Spearman's correlation analysis, we found that the expression of miR-152 was inversely correlated with 
A
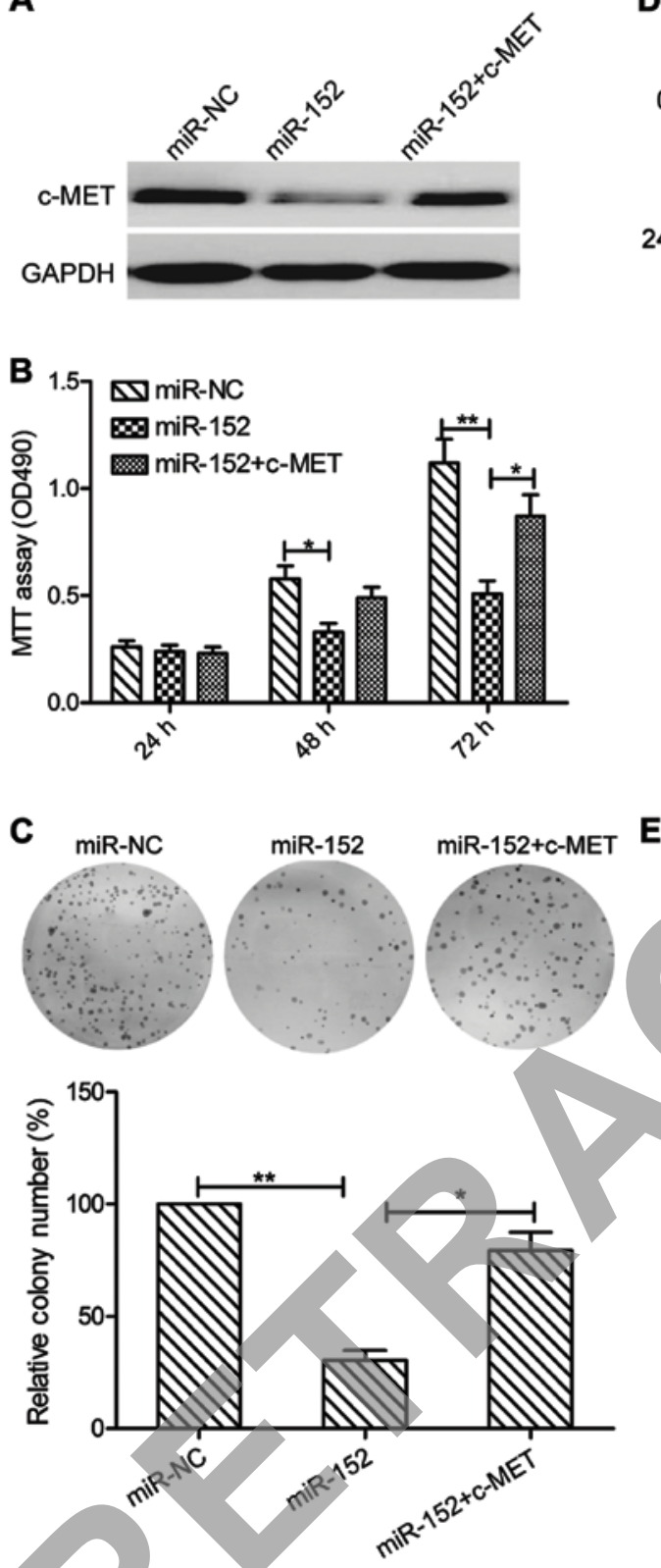

$\mathbf{E}$
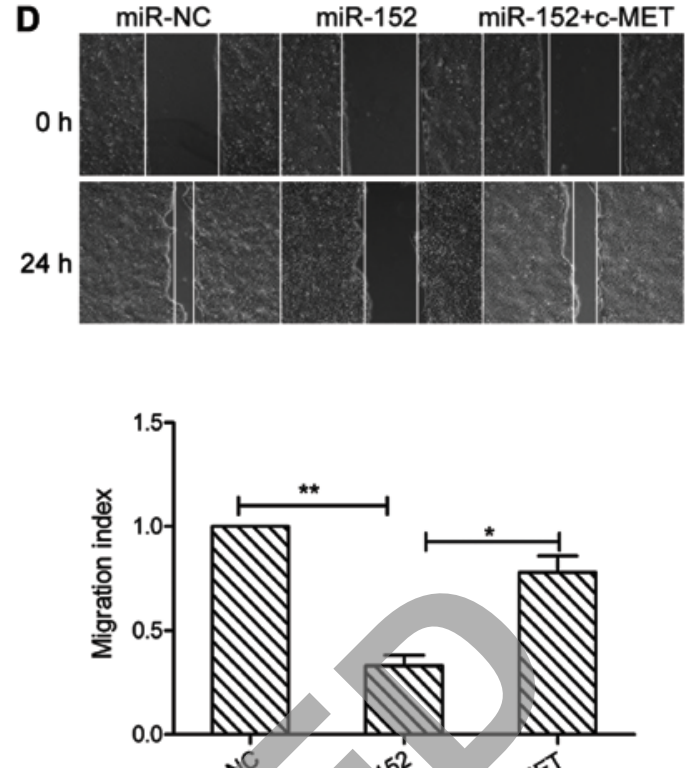

0.0
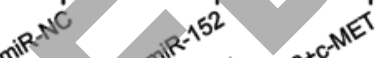
miR-NC miR-152 miR-152+c-MET
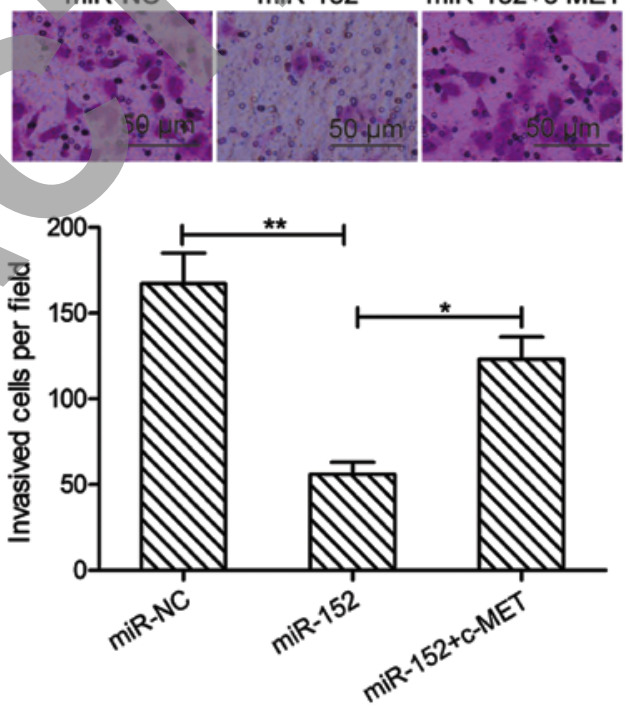

Figure 5. Overexpression of c-MET reverses the tumor suppressive effect of miR-152 in OSCC. (A) c-MET protein expression was determined in SCC9 cells transfected with miR-NC and miR-152 mimic with/without c-MET overexpression plasmid by western blotting. GAPDH was used as the internal control. (B-E) Cell proliferation, colony formation, migration and invasion were detected in SCC9 cells transfected with miR-NC or and miR-152 mimic with/without c-MET overexpression plasmid. ${ }^{*} \mathrm{P}<0.05,{ }^{* *} \mathrm{P}<0.01$.

$c-M E T$ mRNA expression in the 40 patients with OSCC ( $\mathrm{r}=-0.512 ; \mathrm{P}=0.001 ;$ Fig. 4B).

Overexpression of $c$-MET reverses the tumor suppressive effect of miR-152 in OSCC. To evaluate if c-MET is responsible for the functional effects of miR-152 in OSCC cells, SCC9 cells were transfected with miR-NC, miR-152 mimic, or cotransfected with miR-152 mimic and c-MET overexpression plasmid, respectively. Subsequently, cell proliferation, colony formation, migration and invasion were determined in the above cells. As indicated in Fig. 5A, the protein expression of c-MET was increased in the group of cells cotransfected with miR-152 mimic and c-MET plasmid, compared with that transfected only with miR-152 mimic. In addition, c-MET overexpression could partially counteract the decrease of cell proliferation, colony formation, migration and invasion and migration of SCC-9 cells by miR-152 induction (Fig. 5B-E). These results indicated that the suppressive effect of miR-152 on OSCC cells may be via inhibition of $c-M E T$.

miR-152 suppresses tumorigenesis of OSCC in vivo. To further test the effect of miR-152 on tumorigenesis of OSCC in vivo, a xenograft mouse model was established by subcutaneous injection of SCC-9 cells transfected with miR-NC or miR-152 mimic, and tumor growth was measured. The growth curve revealed that miR-152 overexpression group exhibited significantly reduced tumor growth compared with miR-NC group (Fig. 6A). At day 30 post-injection, the mice were sacrificed, and tumor tissues were removed and weighed. miR-152 overexpression group had smaller size (Fig. 6B) and weight 
A
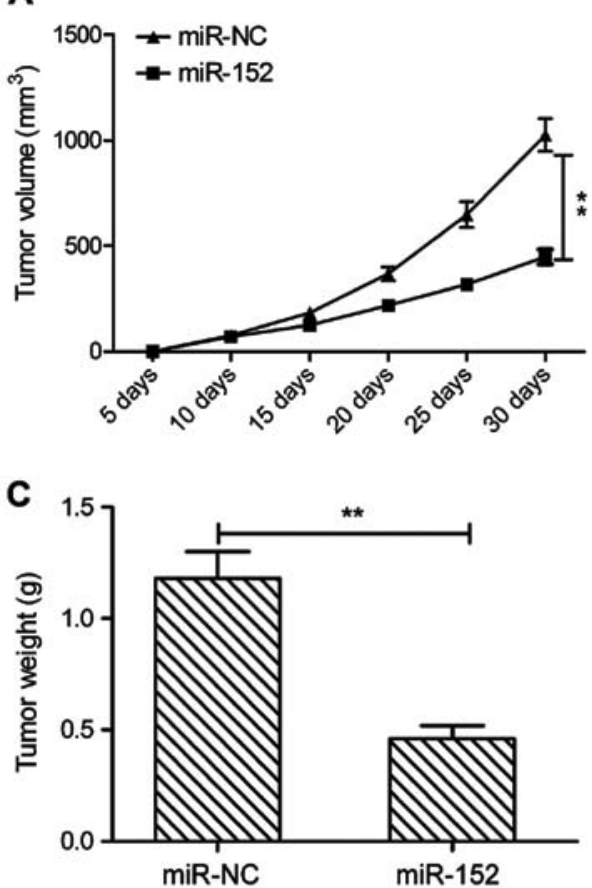

B

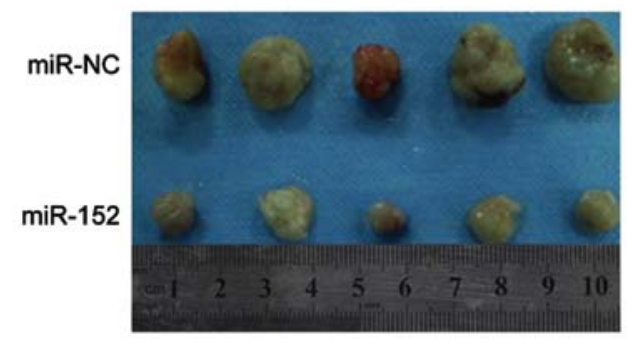

D

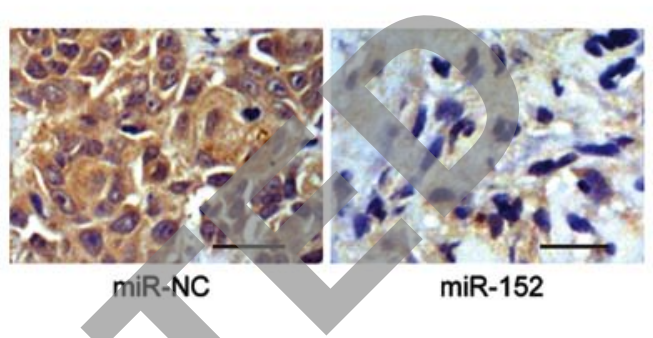

Figure 6. miR-152 suppresses tumorigenesis of OSCC in vivo. (A) Tumor growth was measured every 5 days until the mice were sacrificed. (B) The mice were sacrificed at 30 days after inoculation, and the tumor tissues were imaged. (C) Tumor weight in the different groups. (D) c-MET expression was determined in xenograft tumors by immunohistochemistry. ${ }^{*} \mathrm{P}<0.05,{ }^{* *} \mathrm{P}<0.01$.

(Fig. 6C) than that of miR-NC group. In addition, we also found that c-MET expression was weaker in tumor tissues of miR-152 compared to miR-NC group by immunohistochemistry. These results implied that miR-152 suppressed tumor growth of OSCC in vivo by repressing C-MET.

\section{Discussion}

It was well known that the development and progression of OSCC is a very complicated process that involves the aberrant expression of number of genes and alterations of multiple signaling pathways $(19,20)$. miRNAs have been reported to play crucial roles in the development and progression of OSCC through regulating various target genes $(9,10)$, thus, exploring novel miRNAs in OSCC is crucially important for developing diagnosis marker and therapy agent. In this study, we were interested in the role of miR-152 in OSCC progression.

miR-152, a member of miR-148/152 family, has been reported to be downregulated, and to function as a tumor suppressive miRNA in majority types of cancer (11-16). In contrast, in neuroblastoma and nasopharyngeal carcinoma, miR-152 expression was upregulated, suggesting that miR-152 acts as an oncogene miRNA $(21,22)$. These results suggested that miR-152 could serve as either oncogene or tumor suppressor in different types of cancer. Here, we investigated the expression of miR-152 in OSCC tissues and cell lines, and found that miR-152 expression was downregulated in OSCC cell lines and tissues, and decreased miR-152 was associated with lymph node metastasis and overall disease-free survival of patients. Function assay demonstrated that miR-152 overexpression suppressed OSCC cell proliferation, colony formation, migration and invasion in vitro, as well as inhibited OSCC growth in nude mouse model. These results implied that miR-152 function as a tumor suppressor miRNA in OSCC.

It has been shown that miR-152 inhibits the proliferation and metastases of tumors via repressing multiple genes, such as B7-H1 (23), PIK3CA (24), WNT-1 (15), ALCAM (13), RTKN (25), neuropilin-1 (14) and PIK3R3 (12), all of which are known as important mediators in tumorigenesis or metastasis in enhancing cell proliferation, survival, migration, invasion capacity or disturbing normal cell generation cycle, as well as inducing cell apoptosis. Here, cellular-mesenchymal to epithelial transition factor (c-MET) is indentified to be another important target of miR-152 by luciferase reporter assay, qRT-PCR and western blotting. c-MET, a receptor for hepatocyte growth factor (HGF), has been reported to promote tumorigenicity in a variety of cancers by increasing cellular proliferation, vascular invasion, epithelial-mesenchymal transi-tion, and migration or invasion capacity as well as decreasing apoptosis (25-27). It has been showed that c-MET exerts its biological role by activation of downstream signal transducer molecules, such as protein kinase B, ERK, and signal transducer and activator of transcription 3 (STAT3), PI3K/AKT, and Wnt signaling pathway (28-30). These studies indicated that inhibition of c-MET and its downstream signaling could be the potential strategy for treatment of cancers. In OSCC, c-MET has been shown to be upregulated in OSCC tissues, and its expression was associated with tumor stage (31). In addition, downregulating of c-MET could inhibit OSCC cell proliferation and invasion (32). Thus, in our study, we selected c-MET as a direct target of miR-152, and found that overexpression miR-152 significantly inhibited c-MET and the downstream signaling pathway, c-MET expression was upregulated in OSCC tissues, and inversely correlated with 
miR-152 expression. Of note, we found that restoring c-MET expression attenuated miR-152 induced inhibitory effects in OSCC cells. In vivo study confirmed that restoration of miR-152 suppressed tumor growth in xenograft nude mice by repressing $c$-MET. Collectively, these findings demonstrated that miR-152 exerts suppressive role in OSCC, at least in part, by repressing c-MET.

Some limitations in this study should be considered. First, the function of miR-152 was evaluated through overexpression of miR-152 in a gain-of-function model. Loss-of-function studies via downregulation of miR-152 in OSCC cell lines are needed to verify our findings. Second, using two or more cell lines are needed to validate the role of miR-152 in tumor development and progression. Third, the miR-152 has multiple target genes which could influence the progression and metastases of OSCC, it is anticipated that more molecular mechanisms would be uncovered in our future study.

Taken together, this study showed that miR-152 expression was downregulated in OSCC cell lines and tissues, and low expression is associated with lymph node metastasis and the overall disease-free survival of patients. Further investigation revealed that overexpression of miR-152 inhibited cell proliferation and colony formation, migration and invasion, as well as suppressed tumor growth in vivo by repressing c-MET. These results indicate that miR-152 may serve as a useful therapeutic strategy for OSCC.

\section{References}

1. Siegel R, Ma J, Zou Z and Jemal A: Cancer statistics, 2014. CA Cancer J Clin 64: 9-29, 2014

2. Liao CT, Hsueh C, Lee LY, Lin CY, Fan KH, Wang HM, Huang SF, Chen IH, Kang CJ, Ng SH, et al: Neck dissection field and lymph node density predict prognosis in patients with oral cavity cancer and pathological node metastases treated with adjuvant therapy. Oral Oncol 48: 329-336, 2012.

3. Jerjes W, Upile T, Petrie A, Riskalla A, Hamdoon Z, Vourvachis M, Karavidas K, Jay A, Sandison A, Thomas GJ et al: Clinicopathological parameters, recurrence, locoregional and distant metastasis in 115 T1-T2 oral squamous cell carcinoma patients. Head Neck Oncol 2: 9, 2010.

4. Bartel DP: MicroRNAs: Genomics, biogenesis, mechanism, and function. Cell 116: 281-297, 2004.

5. Malan-Müller S, Hemmings SMand Seedat S: Big effects of small RNAs: A review of microRNAs in anxiety. Mol Neurobiol 47: 726-739, 2013.

6. McManus MT: MicroRNAs and cancer. Semin Cancer Biol 13: 253-258, 2003.

7. Farazi TA, Spitzer JI, Morozov P and Tuschl T: miRNAs in human cancer. J Pathol 223: 102-115, 2011.

8. Garzon R and Marcucci G: Potential of microRNAs for cancer diagnostics, prognostication and therapy. Curr Opin Oncol 24: 655-659, 2012.

9. Prasad G, Seers C, Reynolds E and McCullough MJ: A panel of microRNAs can be used to determine oral squamous cell carcinoma. J Oral Pathol Med 46: 940-948, 2017.

10. Ries J, Baran C, Wehrhan F, Weber M, Neukam FW, Krautheim-Zenk A and Nkenke E: Prognostic significance of altered miRNA expression in whole blood of OSCC patients. Oncol Rep 37: 3467-3474, 2017.

11. Xie G, Li W, Li R, Wu K, Zhao E, Zhang Y, Zhang P, Shi L, Wang D, Yin Y, et al: Helicobacter pylori promote B7-H1 expression by suppressing miR-152 and miR-200b in gastric cancer cells. PLoS One 12: e0168822, 2017.
12. $\mathrm{Li} \mathrm{B}, \mathrm{Xie} \mathrm{Z}$ and $\mathrm{Li} \mathrm{B}$ : miR-152 functions as a tumor suppressor in colorectal cancer by targeting PIK3R3. Tumour Biol 37: 10075-10084, 2016.

13. Chen MJ, Cheng YM, Chen CC, Chen YC and Shen CJ: miR-148a and miR-152 reduce tamoxifen resistance in $\mathrm{ER}^{+}$breast cancer via downregulating ALCAM. Biochem Biophys Res Commun 483: 840-846, 2017.

14. Zhang YJ, Liu XC, Du J and Zhang YJ: miR-152 regulates metastases of non-small cell lung cancer cells by targeting neuropilin-1. Int J Clin Exp Pathol 8: 14235-14240, 2015.

15. Tang XL, Lin L, Song LN and Tang XH: Hypoxia-inducible miR-152 suppresses the expression of WNT1 and ERBB3, and inhibits the proliferation of cervical cancer cells. Exp Biol Med (Maywood) 241: 1429-1437, 2016.

16. Zhu C, Li J, Ding Q, Cheng G, Zhou H, Tao L, Cai H, Li P, Cao Q, Ju X, et al: miR-152 controls migration and invasive potential by targeting TGF $\alpha$ in prostate cancer cell lines. Prostate 73: 1082-1089, 2013

17. Li B, Yang XX, Wang D and Ji HK: MicroRNA-138 inhibits proliferation of cervical cancer cells by targeting c-Met. Eur Rev Med Pharmacol Sci 20: 1109-1114, 2016.

18. Chau L, Jabara JT, Lai W, Svider PF, Warner BM, Lin HS, Raza SN and Fribley AM: Topical agents for oral cancer chemoprevention: A systematic review of the literature. Oral Oncol 67: $153-159,2017$

19. Gharat SA, Momin M and Bhavsar C: Oral squamous cell carcinoma: Current treatment strategies and nanotechnologybased approaches for prevention and therapy. Crit Rev Ther Drug Carrier Syst 33: 363-400, 2016.

20. Jasinski-Bergner S, Stoehr C, Bukur J, Massa C, Braun J, Hüttelmaier S, Spath V, Wartenberg R, Legal W, Taubert H, et al: Clinical relevance of miR-mediated HLA-G regulation and the associated immune cell infiltration in renal cell carcinoma. OncoImmunology 4: e1008805, 2015.

1. Liu DZ, Ander BP, Tian Y, Stamova B, Jickling GC, Davis RR and Sharp FR: Integrated analysis of mRNA and microRNA expression in mature neurons, neural progenitor cells and neuroblastoma cells. Gene 495: 120-127, 2012.

22. Huang S, Li X and Zhu H: MicroRNA-152 Targets phosphatase and tensin homolog to inhibit apoptosis and promote cell migration of nasopharyngeal carcinoma cells. Med Sci Monit 22: 4330-4337, 2016.

23. Wang Y, Wang D, Xie G, Yin Y, Zhao E, Tao K and Li R: MicroRNA-152 regulates immune response via targeting B7-H1 in gastric carcinoma. Oncotarget 8: 28125-28134, 2017.

24. Ge S, Wang D, Kong Q, Gao W and Sun J: Function of miR-152 as a tumor suppressor in human breast cancer by targeting PIK3CA. Oncol Res 25: 1363-1371, 2017.

25. Zhou J,Zhang Y, Qi Y, Yu D, Shao Q and Liang J: MicroRNA-152 inhibits tumor cell growth by directly targeting RTKN in hepatocellular carcinoma. Oncol Rep 37: 1227-1234, 2017.

26. Skead G and Govender D: Gene of the month: MET. J Clin Pathol 68: 405-409, 2015

27. Marano L, Chiari R, Fabozzi A, De Vita F, Boccardi V, Roviello G, Petrioli R, Marrelli D, Roviello F and Patriti A: c-Met targeting in advanced gastric cancer: An open challenge. Cancer Lett 365: 30-36, 2015.

28. Boccaccio C, Luraghi P and Comoglio PM: MET-mediated resistance to EGFR inhibitors: An old liaison rooted in colorectal cancer stem cells. Cancer Res 74: 3647-3651, 2014

29. Ho-Yen CM, Jones JL and Kermorgant S: The clinical and functional significance of c-Met in breast cancer: A review. Breast Cancer Res 17: 52, 2015.

30. Mazzone M and Comoglio PM: The Met pathway: Master switch and drug target in cancer progression. FASEB J 20: 1611-1621, 2006.

31. Freudlsperger C, Alexander D, Reinert S and Hoffmann J: Prognostic value of c-Met expression in oral squamous cell carcinoma. Exp Ther Med 1: 69-72, 2010.

32. Yasui H, Ohnishi Y, Nakajima M and Nozaki M: Migration of oral squamous cell carcinoma cells are induced by HGF/c-Met signalling via lamellipodia and filopodia formation. Oncol Rep 37: 3674-3680, 2017. 